\title{
Transient Squeal Analysis of a Non Steady State
}

\section{Manoeuvre}

\author{
Oliver Stump ${ }^{1,2}$, Maximilian Könning ${ }^{1}$ and Wolfgang Seemann ${ }^{2}$ \\ 1. Daimler AG, Group Research \& MBC Development, Sindelfingen 71059, Germany \\ 2. Institute of Engineering Mechanics-Karlsruhe Institute of Technology (KIT), Karlsruhe 76131, Germany
}

\begin{abstract}
Brake squeal is one of the main NVH (vibration harshness) challenges in the brake development of passenger cars. The conflict of goals in the development process and the late testability leads to the need of a deeper basic understanding of the squeal phenomenon and definition of design rules. On the other hand, brake squeal is still a very interesting field of research also for the universities because of its combination of different fundamentals, such as friction and stability behaviour of systems with local nonlinearities. Major nonlinearities of the brake system are the joints, especially the contact areas formed by the oscillating brake pad and the caliper. The state-of-the-art calculation method, which still is the "complex eigenvalue analysis", linearizes these joints, hence, neglecting its nonlinear influence in the stability analysis. Vehicle and bench experiments show that special driving manoeuvres like parking, where the brake pad often leaves the steady state, are likely causing brake squeal. The system in these manoeuvres sometimes behaves opposed to the linearized stability analysis, indicating a limit cycle beyond the Hopf point. Therefore, these states must be investigated more closely. This paper investigates the nonlinear influence of the pad caliper joint in a fixed brake caliper, also called abutment. Bench tests with pressure foils at the abutment of the brake caliper and mode shape analysis were done and a simple FE (finite element) model for a transient simulation is proposed. It is shown that the joint activity varies with driving manoeuvres, leading to different stability behaviours and limiting cycle amplitudes.
\end{abstract}

Key words: Brake squeal, transient analysis, nonlinear joint, stability analysis, finite element method.

\section{Introduction and Previous Work}

The NVH requirements for passenger cars are high and are probably going to increase with the ongoing electrification of the power train. In addition, the customer can nowadays also be standing outside of the vehicle, e.g. parking his car remotely. Brake squeal is still one of the main NVH phenomena at the vehicle brake, challenging both, the bench/vehicle testing and the simulation departments. For the bench tests many test procedures and sequences have been developed to test the different states of the brake and indices have been created to evaluate the occurrences, amplitudes and frequency of the squeal [1]. Also vehicle testers develop a "feeling" for the brake, which leads to critical working points that often create

Corresponding author: Oliver Stump, M.Sc., research fields: method development and simulation for brake development of passenger cars. squeals even though the brake is silent in the rest of the test drive. One of this manoeuvres is parking (without cornering), which was linearly analysed in Ref. [2] and this analysis shall be continued with nonlinear methods in this work.

From the simulation side large effort is carried out to tackle the squeal problem in general. Despite its known limits, the linear method of calculating the "complex eigenvalues" of the system is still the state of the art. Because of its fast calculation time many working points can be investigated leading to a good overview of the robustness of the brake [3]. Unfortunately, the brake system is highly nonlinear [4-7] and linear stability analysis can lead to wrong results. Not only the amplitudes of the limit cycles cannot be calculated but also subcritical flutter can occur, meaning that with the right initial conditions a stable limit cycle beyond the linear stability borders can be found. Oberst et al. [6] showed in his report 
that if one solely considers the nonlinearity of the pad and the disc contact subcritical flutter can already occur. Kruse et al. [5] showed in a minimal model, that the joints e.g. the contact between brake pad and caliper, play a significant role and also can lead to subcritical flutter of the system. In the following investigation, the change in direction of the brake disk velocity while braking is examined. This will be called parking. The effects of cornering are not part of this work.

\section{Pressure Foil Measurements}

The main mechanism while parking is the movement of the brake pad in the caliper. To qualitatively understand how the brake pad gets in contact with the caliper after a change of direction of the car, pressure foil measurements on a real brake system are done between the brake pad and the caliper. The sampling rate of the pressure foil is $100 \mathrm{~Hz}$, hence, too low to see the oscillation of the squealing. But as seen in Fig. 1, the change in the contact surfaces of the abutment area can be observed. Fig. 1 shows two

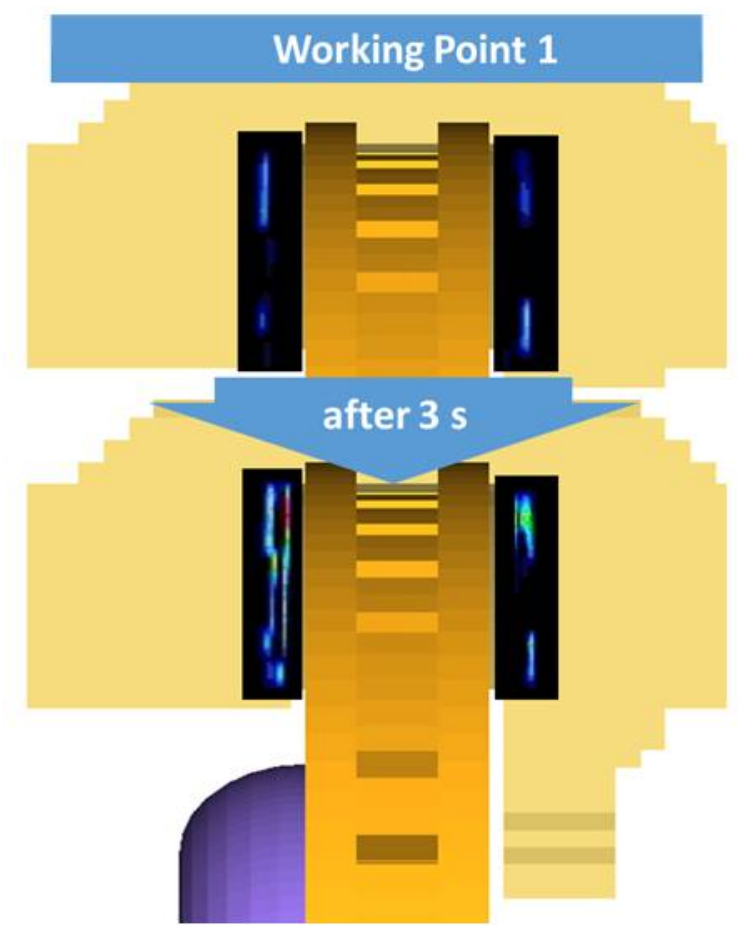

Fig. 1 Pressure foil measurements at two working points with the same pad at the beginning of the brake application and a certain time interval later. The foil results are embedded into mesh data for better clarity. different low pressure brake applications exactly after a change of direction of the disk. The same pad/disk configuration at different pressure levels and temperatures is used. The brake pads touch the abutment at the beginning of the parking brake manoeuvre and then the contact area increases over time until a steady state is reached. The increase in contact area is not the same for every working point, due to e.g. the application point of the friction force and is also dependant on production tolerances, especially at the edge of the back plate. The inner and outer pad behaves differently, which can be explained with the deformation of the one side mounted caliper.

More measurements are done with different working points and different pads. Because of the mentioned variability and the influences of the production tolerances, the pressure foil measurements can only lead to a better understanding of the parking process, rather than to validate the simplified FE simulation. The measurements motivate the simulation of a variable abutment surface and its influence onto the squeal probability. This was already done in Ref. [2].

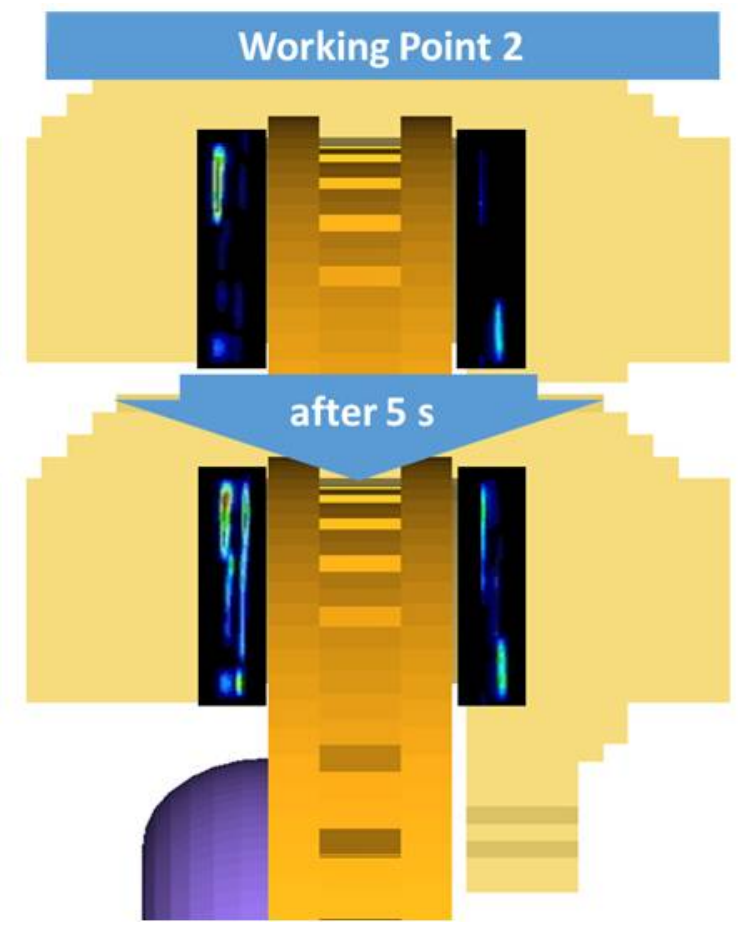


The abutment contacts between inner and outer pad could be different, which can also be included in a linear stability analysis.

Furthermore, the measurements of Fig. 1 motivate a nonlinear analysis of the process. As shown in Ref. [5] the nonlinear joint at the abutment can lead to a subcritical flutter. Assuming that the change in the contact states shown in Fig. 1 can be simplified as a change in the axial stiffness of the minimal model in Ref. [5], during a parking manoeuvre the system has a higher probability to hit the critical area between a Fold point and a Hopf point. In this area, stable limit cycles can occur, but the system does not need to show an instability in the linearization around the equilibrium. In addition the "hit" of the pad from leading to trailing edge, represents a large change in the initial conditions, which also increases to probability to reach the zone of attraction of a stable limit cycle.

\section{Linear and Nonlinear Stability Analysis}

To analyse the nonlinear stability behaviour of the FE brake model introduced in Ref. [2], nonlinear transient simulations are carried out. The linear stability is shown in Fig. 2. The stability border for the $2 \mathrm{kHz}$ instability is either around $\mu_{\text {crit }} \approx 0.8$ for the axially free abutment or with a fixed abutment around $\mu_{\text {crit }} \approx 1.0$. The same system parameters will be used for the nonlinear transient simulation, except for the friction value $\mu$ between disk and pad, which will be $\mu$ $=0.4$, so no stable limit cycle can be anticipated, based on the linear stability analysis.

\subsection{Model Discussion: Nonlinearities in the System}

There are two main nonlinearities in the model: the contact between disk and pad and the contact between pad and caliper, the abutment, as shown in Fig. 3. As shown in Ref. [6] the pad disk contact solely can be enough to introduce subcritical flutter. However, for this investigation, the movement of the pad between leading and trailing edge has to be simulated, and therefore contacts at the edges of the pad are necessary. Another nonlinearity could be the piston to caliper contact or piston to back plate contacts. In the presented study these contacts are neglected, but they should be investigated in future work.

\subsection{Model Discussion: Damping and Numerical Damping}

As discussed by many authors, the brake squeal phenomena is very sensitive to damping $[4,8]$. But despite the clear need, the mechanisms and implementation of damping are still not fully understood. Hence, in this study no material damping is used. Only the damping resolving from the friction force and the numerical damping of the dynamics of

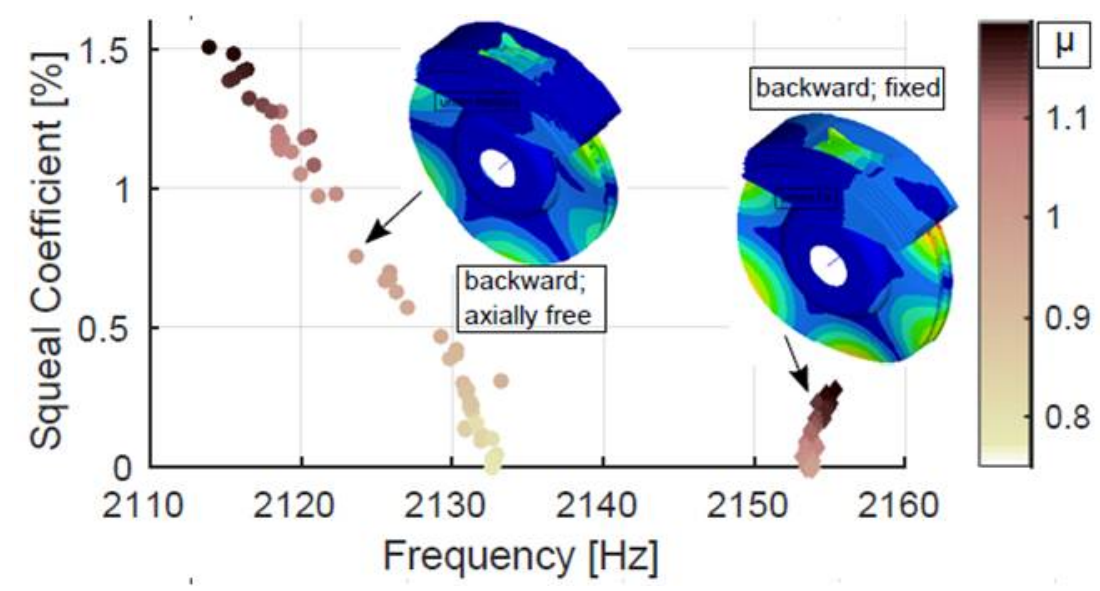

Fig. 2 Linear stability analysis of working points with increasing $\mu$. With an axially free abutment the $2 \mathrm{kHz}$ instability appears at around $\mu_{\text {crit }} \approx 0.8$ and with fixed abutment around $\mu_{\text {crit }} \approx 1.0$. 
the solver is present. The parameters of the solver will then be tuned, so that the results are matching the bench tests. This again shows the difficulty in predicting the squeal probability of an unknown brake system without available hardware at this stage of the development.
For this study, the implicit HHT (Hilber-Hughes-Taylor $\alpha$-Method) is used in Abaqus. The solver dynamic is dependent on the numerical parameter $\alpha$, the time step and the angular frequency under investigation [9]. Fig. 4 shows the results of three different $\alpha$ values. For $\alpha=0$ (a) many



Fig. 3 Model overview and zoom into the two nonlinearities of the transient model.
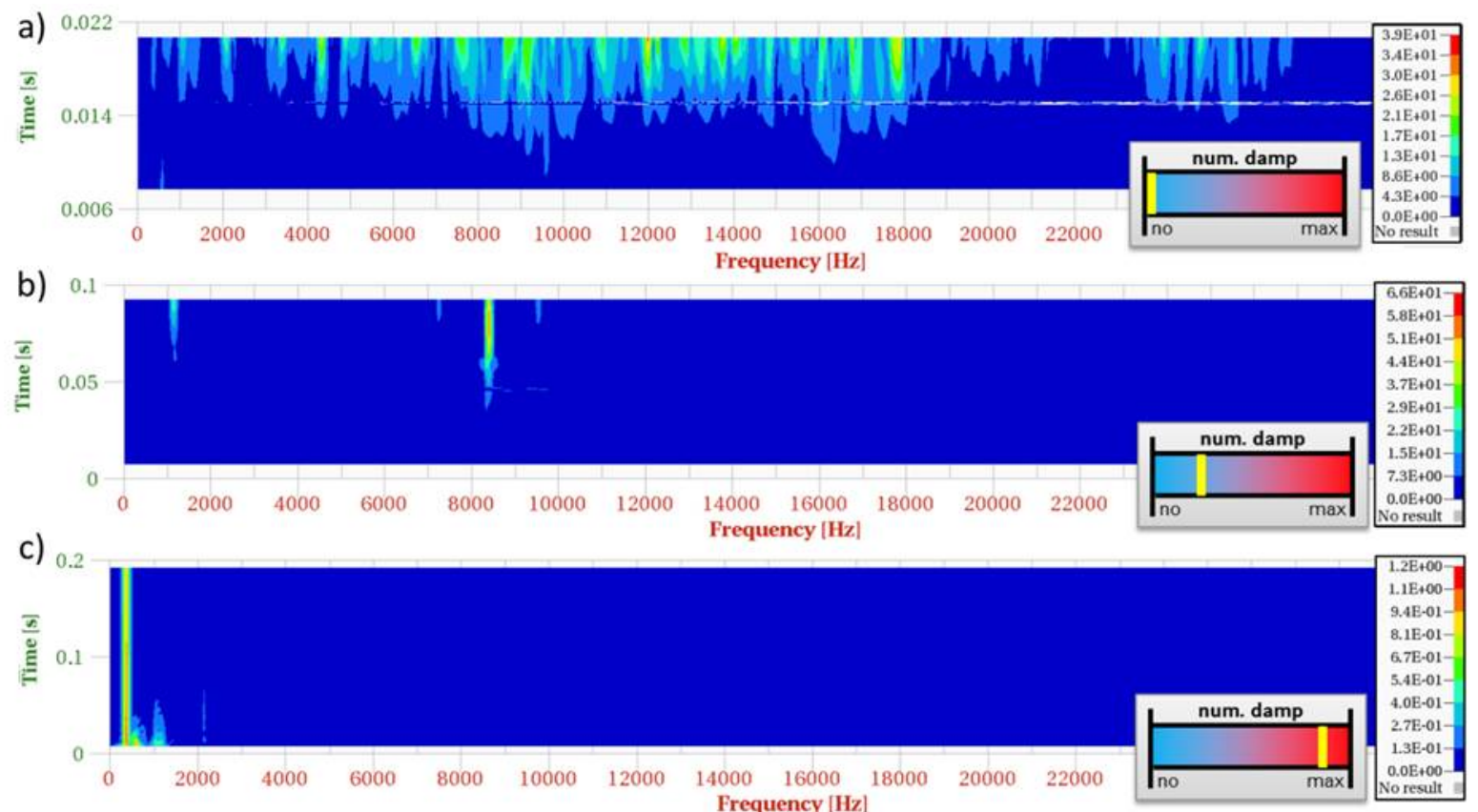

Fig. 4 Waterfall diagram of the velocity of a disk node for the transient results with different numerical damping. (a) $\alpha=0$, (b) $\alpha=-\mathbf{0 . 1}$ and (c) $\alpha=\mathbf{- 0 . 4 1 4 2 1}$ (abaqus default value for contact problems). Colour scale of the plots is different. 
frequencies occur, which is unrealistic. With small numerical damping (b) a $9 \mathrm{kHz}$ instability arises, which cannot be seen in the higher damped simulation (c).

Because of the described unknown system damping, one can a priori not judge, if the lesser damped result is representing a real squeal. At the bench tests, with the underlying real brake system, a $2 \mathrm{kHz}$ and a $9 \mathrm{kHz}$ squeal were observed. Hence, the lower damped simulation could represent a real squeal scenario, even though this study focuses on the low frequency squeal. If not mentioned otherwise the "high" numerical damping will be used in the following.

\subsection{Nonlinear Transient Results}

The goal of the nonlinear transient simulation is to compare it with the linearized results and to simulate different pad movements and their influence to the resulting limit cycle. As shown in Fig. 2 the critical $\mu$ of the used working point is $\mu_{\text {crit }} \approx 0.8$. Hence, the system should not show any stable limit cycles at this frequency range, assuming the linearization state is correct. In Figs. 5a and 5b waterfall diagrams of the velocity of a disk node are shown. The transient simulation starts with a perfectly aligned pad for the forward and backward direction, so there is no pad movement necessary to establish contact with the abutment. Initially one can observe a $2 \mathrm{kHz}$ vibration, but it is damped out over time, which correlates to the linear analysis.

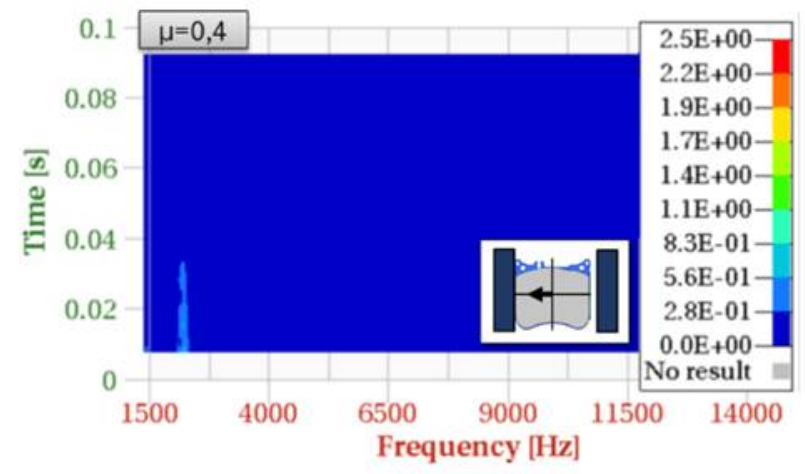

(a)
Fig. 6 shows the waterfall diagram of the same node of the mesh, but with initially a gap between pad and caliper. The gap leads to a hit of the pad against the caliper causing the critical initial conditions for a stable $2 \mathrm{kHz}$ limit cycle. With no tested linearization state this instability could be found. Hence, the system here has a subcritical Hopf bifurcation. Based on the experiences from bench and vehicle tests, this could highlight the criticality of the parking manoeuvre. Not only the brake experiences different contact states, but the manoeuvre itself also leads to critical initial conditions for a subcritical flutter.

\subsection{Comparisons of the Linearized Mode Shapes with the Transient Results}

To get a deeper understanding of the differences of the linear and nonlinear results the mode shape of the linearized system and the ODS (operating deflection shape) of the stable limit cycle is compared. In the macroscopic comparison in Fig. 7 the accelerations look nearly the same of the linearized and nonlinear transient results. The main differences obviously lie in the local nonlinearities.

The resulting limit cycle of the transient simulation in this specific working point is very dominant, hence the results are directly comparable to the linear mode shapes. Fig. 8 shows the acceleration of the transient result at $0.199 \mathrm{~s}$. The deformation scale is increased to see the relative movement at the abutment. Even though the main motion of the trailing side of the pad

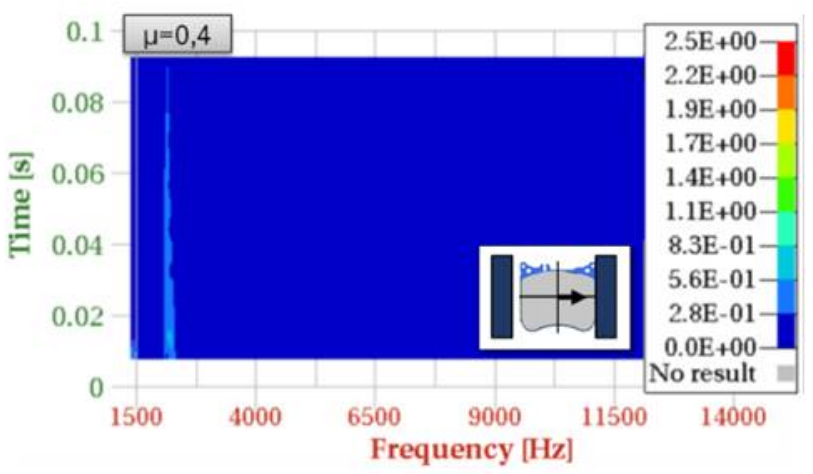

(b)

Fig. 5 Waterfall diagram of the nonlinear transient analysis starting with a perfectly aligned pad. Displayed is the velocity of a disk node. 


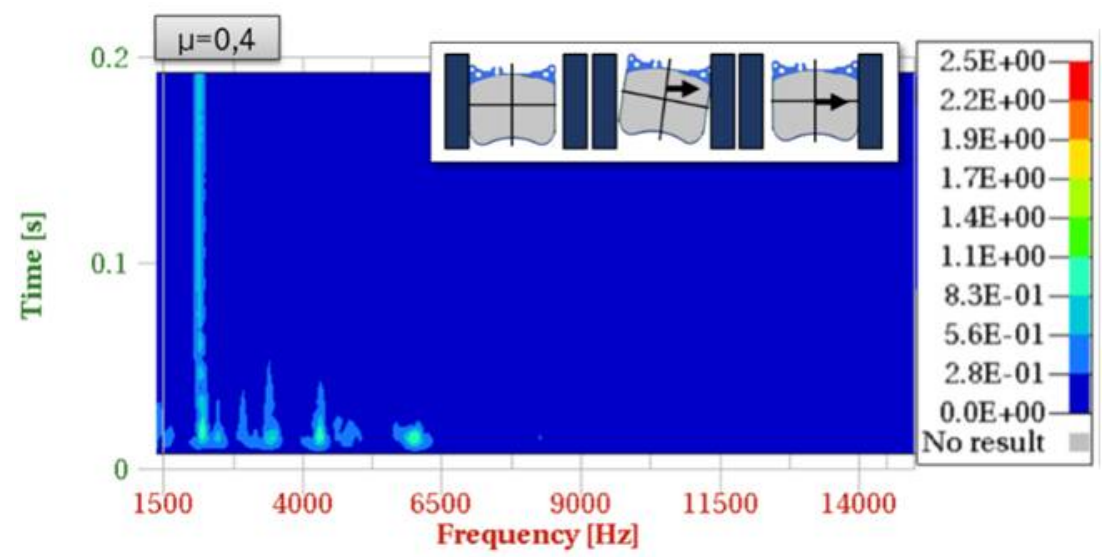

Fig. 6 Waterfall diagram of the nonlinear transient analysis starting with a gap between pad and caliper. Displayed is the velocity of a disk node.

a)

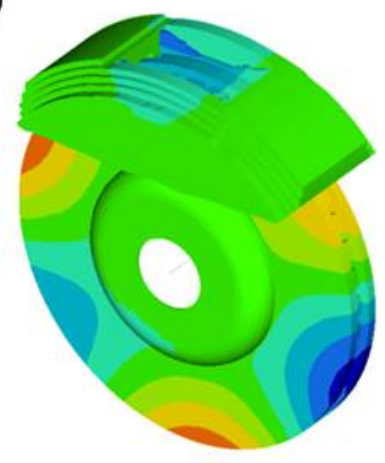

b)

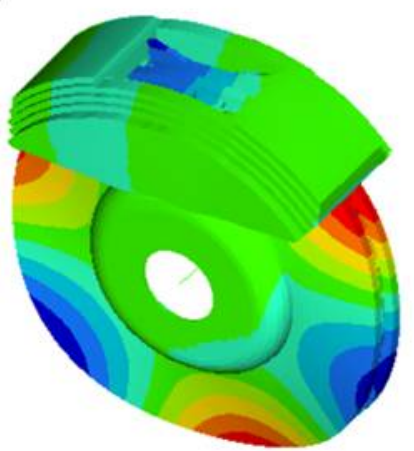

c)

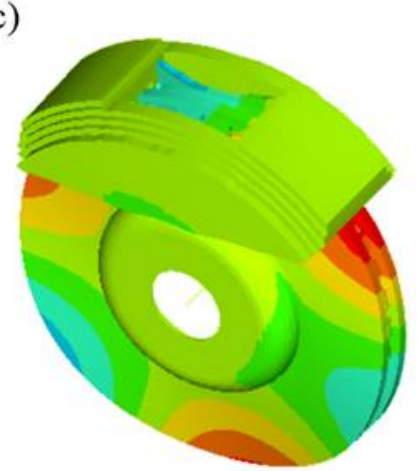

Fig. 7 Nonlinear transient momentum state at $\mathbf{t}=\mathbf{0 . 1 9 9} \mathrm{s}$ showing only the axial acceleration (a). Complex mode shapes of two different linearizations at a close frequency (b) and (c) for comparison.



Fig. 8 Scaled acceleration of the nonlinear transient result at $t=0.199 \mathrm{~s}$. 
in this case is a rotation around the contact line at the abutment in Fig. 8, there is also a small relative movement visible. Hence, in one cycle of the transient oscillation this joint behaves nonlinearly and switches from sliding to sticking. Furthermore, the contacts between disk and pad open and close in certain areas, which introduces another nonlinearity in one cycle. These results again highlight the importance of a nonlinear analysis to investigate brake squeal and to closely look at the joint activity.

The often used MAC (modal assurance criterion), to compare two mode shapes or ODS, can lead to false security. E.g. comparing the two linear mode shapes in Fig. 7 the MAC value is high. This has its origin in the similar macroscopic deformation at that frequency. But as seen in the transient results, small local nonlinearities can change the system behaviour significantly.

Fig. 9 shows the comparison of the displacements of two caliper nodes at the abutment and their corresponding back plate nodes. At the outer pad, sticking can be observed. The inner pad shows a more dominant $2 \mathrm{kHz}$ oscillation. Thus, sticking phases are harder to detect. These exemplary results show the high joint activity at the abutment. Also differences between inner and outer pad can be seen, which corresponds to bench results at low pressure.

\subsection{Excitations to Reach the Observed Limit Cycle}

The presented system has at least one stable limit cycle branch beyond the Hopf point as seen in the results of Fig. 6. Motivated from experience in the bench and car tests the movement of the pad and its hit against the abutment provoked the system to jump from the "quiet" solution into a stable $2 \mathrm{kHz}$ limit cycle. In the following, more initial conditions are tested to evaluate the critical initial conditions and to find excitation methods, which lead to less simulation time.

Fig. 10 shows an overview of different initial positions of the pad and the resulting time simulations. It seems that there are more than one stable limit cycle branches at the same frequency. Different amplitudes can be observed, especially when the pad is initially skew.

When the pad is perfectly aligned, an artificial excitation to provoke the $2 \mathrm{kHz}$ limit cycle could not be found and must be investigated in future work.

b)

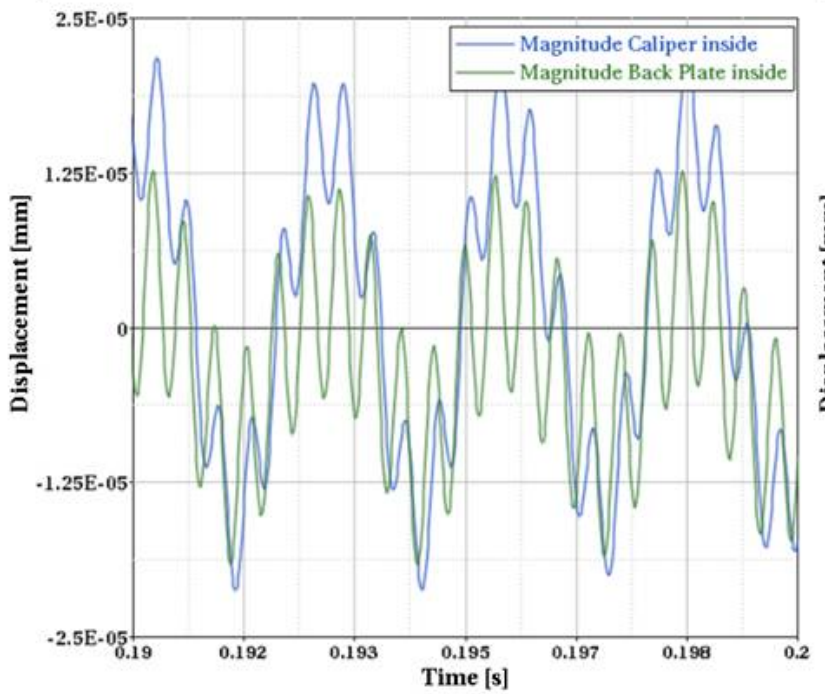

Fig. 9 Comparison of the displacements of two caliper nodes at the abutment and their corresponding back plate nodes. (a) shows exemplary nodes on the inner side of the brake; (b) shows nodes of the outer side; the back plate is mostly sliding at the abutment, but there are also areas where the back plate sticks at the caliper. 

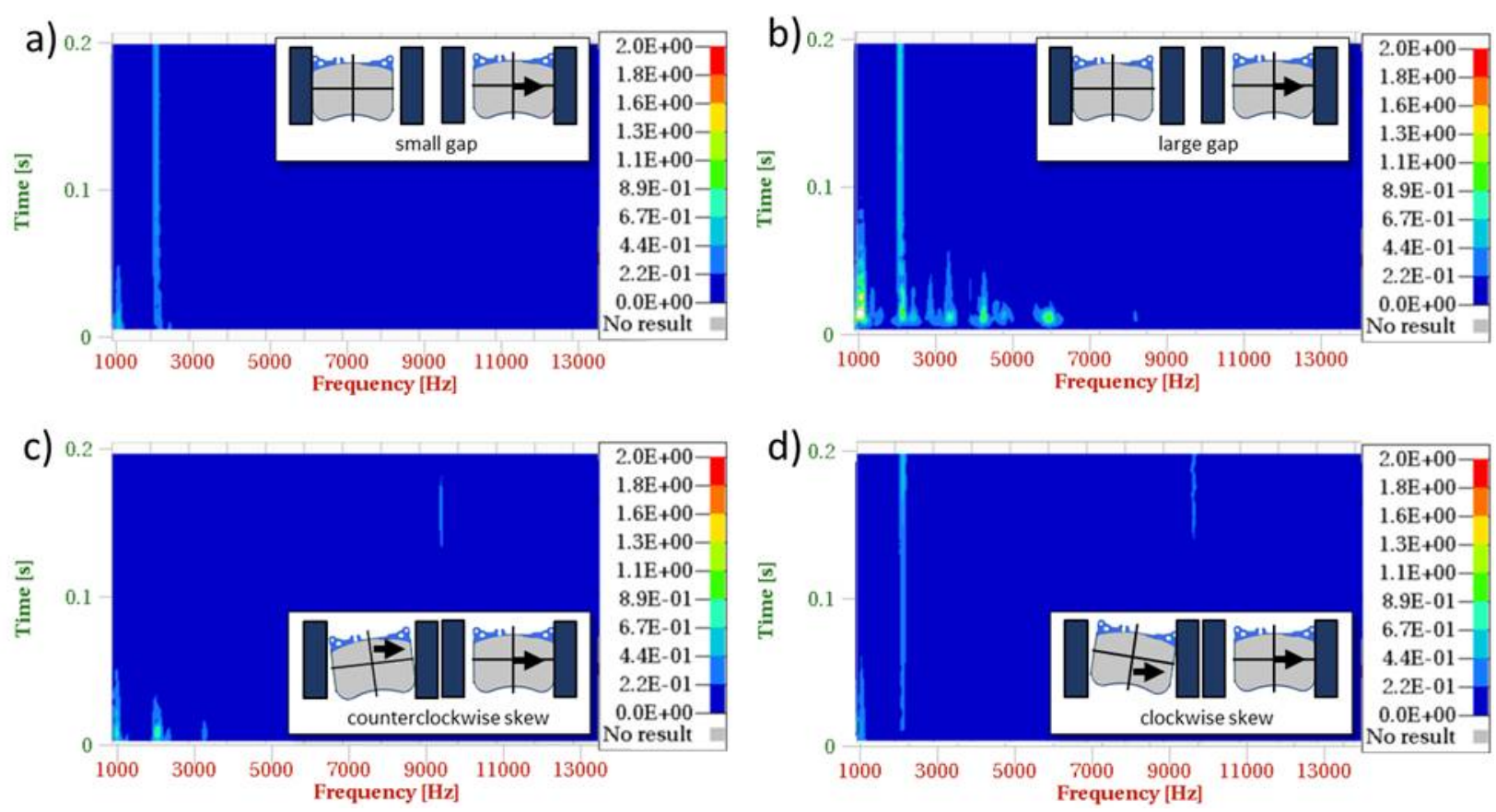

Fig. 10 Nonlinear transient results with different initial conditions. The resulting amplitude of the limit cycle in (a), (b) and (d) is different. In (c) no limit cycle can be found.

\section{Conclusions and Outlook}

This work investigates the nonlinear stability behaviour of a fixed caliper brake system with the load case "the change in direction of the brake disk velocity while braking". The nonlinear transient simulation is motivated by pressure foil measurements at the contact area of the brake pad and the caliper. The measurements show, that the contact area changes widely due to a parking manoeuvre of the vehicle. This validates on one hand the assumptions made in a previous study, in which the linearization points of a complex eigenvalue analysis take the variation of the abutment area into account.

On the other hand, the measurements raise the question, how good the brake system can be simulated with a linear analysis, especially under such manoeuvres.

Subsequently, nonlinear transient simulations are done to investigate the resulting limit cycles of the brake. It is found that at the working point under investigation, the brake system is "quiet" if the brake pad initially is placed perfectly aligned to the caliper.
If the brake pad has initially a gap and hits the caliper, a limit cycle can be observed. With no tested linearization state this instability could be found at the same working point. Like other authors reported before, this indicates, that the nonlinearities of the brake pad can lead to subcritical flutter.

Finally, different excitation and initial states are investigated to reduce the needed simulation time of the nonlinear analysis. It seems that not the axial movement of the brake pad is provoking the limit cycle, but the change in the normal force at the abutment. This could also explain, why parking is such a critical test manoeuvre, because of its large normal force variation.

In future work more effects of the real parking manoeuvre will be included. Additionally, the third nonlinearity of the brake pad, the contact to the piston will be investigated. It would be also very interesting to search for design parameters, which can influence the system in such way, that the subcritical flutter is transformed into a super critical flutter. The stability borders found in a linear analysis would then be valid again. 


\section{Acknowledgements}

This paper was first presented at the proceeding of the EuroBrake2017, May 4, 2017, Germany, under the title EB2017-SVM-004: Transient Squeal Analysis of a Non Steady State Manoeuvre.

\section{References}

[1] Häsler, K., and Bender, A. 2012. "GLONORE-An Approach for a Total Evaluation of Brake Noise." Dresden, EuroBrake 2012

[2] Stump, O., Nunes, R. F., and Seemann, W. 2016. "Brake Squeal: Influence of the Contact Linearization at the Pad Abutment." Milano, EuroBrake 2016.

[3] Nunes, R. F. 2015. "Robustness Analysis of Brake Systems: Build the Bridge between Simulation and Quality Control Tests.” Dresden, EuroBrake 2015.

[4] Hochlenert, D. 2009. "Nonlinear Stability Analysis of a Disk Brake Model.” Nonlinear Dyn. 58 (1): 63-73.
[5] Kruse, S., Tiedemann, M., Zeumer, B., Reuss, P., Hetzler, H., and Hoffmann, N. 2015. "The Influence of Joints on Friction Induced Vibration in Brake Squeal." Journal of Sound and Vibration 340 (31): 239-52.

[6] Oberst, S., and Lai, J. C. S. 2015. "Nonlinear Transient and Chaotic Interactions in Disc Brake Squeal.” Journal of Sound and Vibration 342 (28): 272-89.

[7] Tiedemann, M., Dethlefs, A., Kruse, S., Uhlmann, E., and Hoffmann, N. 2015. "Design of Contact Interfaces to Reduce Noise in Friction Brakes.” Dresden, EuroBrake 2015.

[8] Fritz, G., Sinou, J.-J., Duffal, J. M., and Jezequel, L. 2007. "Effects of Damping on Brake Squeal Coalescence Patterns-Application on a Finite Element Model." Mechanics Research Communications, Elsevier 34 (2): 181-90.

[9] Hilber, H. M., Hughes, T. J. R., and Taylor, R. L. 1977. "Improved Numerical Dissipation for Time Integration Algorithms in Structural Dynamics." Earthquake Engineering and Structural Dynamics 5 (3): 283-92. 